Whitfield et al.: Comparison of different trap designs for capture of noctuid moths

\title{
Comparison of Different Trap Designs for Capture of Noctuid Moths (Lepidoptera: Noctuidae) with Pheromone and Floral Odor Attractants
}

\section{E. Charles Whitfield ${ }^{1 *}$, Enrique Lobos $^{2}$, Alan Cork ${ }^{3}$, and David R. Hall ${ }^{3}$}

${ }^{1}$ The National Institute of Agricultural Botany, East Malling Research, Kent, ME19 6BJ, UK, E-mail: Charles.Whitfield@emr.ac.uk

${ }^{2}$ Facultad de Agronomia y Agroindustrias, Universidad Nacional de Santiago del Estero, Argentina: E-mail: ealobos@gmx.net

${ }^{3}$ University of Greenwich, Natural Resources Institute, Kent, ME4 4TB, UK, E-mail:

D.R.Hall@greenwich.ac.uk

*Corresponding author; E-mail: Charles.Whitfield@emr.ac.uk 
Whitfield et al.: Comparison of different trap designs for capture of noctuid moths

\section{Abstract}

Six trap designs were assessed for capturing noctuid moths in field trials in the UK and Argentina. The traps were baited with either a sex pheromone for Autographa gamma in the UK trials and Helicoverpa gelotopoeon in Argentina, or a floral odor blend. In the UK trials the Universal Trap and a funnel sleeve trap were compared; in Argentina the funnel sleeve trap, a homemade bucket trap, and three sticky traps: LepTrap, wing trap, and delta trap were compared. Comparisons were made between the traps and attractants and captures of noctuid moths and non-target insects. Traps baited with the floral attractant caught a lower number but a wider range of noctuid species including Helicoverpa, Spodoptera, Rachiplusia, Dargida, Mythimna, Chrysodeixis, Agrotis, and Autographa spp, and non-target insects. In the UK trials, the funnel sleeve trap caught significantly more A. gamma than the Universal Trap. The addition of an insecticide to the Universal Trap marginally increased trap catches. In the Argentinian trials, the homemade bucket and the sleeve traps outperformed all sticky traps in most situations regardless of crop environment and attractant type. The homemade bucket and the funnel sleeve traps are also considerably cheaper than the other trap designs. Of the sticky traps the LepTrap caught more noctuids than the wing and delta traps. The results suggest that the bucket trap and the sleeve trap have a much greater maximum capacity and possibly a greater retention efficiency for noctuid moths compared to the sticky traps.

Key words. Trapping, Helicoverpa gelotopoeon, Autographa gamma, kairomone, floral attractant. 
Whitfield et al.: Comparison of different trap designs for capture of noctuid moths

Some of the most economically damaging crop pests belong to the family Noctuidae which includes the armyworms (Spodoptera spp. and Mythimna spp.), bollworms (Helicoverpa spp.), cutworms (Agrotis spp.), as well as the corn earworm (Helicoverpa zea), tobacco budworm (Heliothis virescens), and many other crop pests. Trapping these crop pests is crucial to crop protection programs, whether for pest monitoring to determine the optimum time to apply other control agents or for mass trapping.

There are numerous types of traps available for catching pest moth species in combination with an attractant. The shape, size of opening, method of retaining the insects, capacity, and cost varies amongst these traps. Certain design features are likely to impact on how successful a trap is at catching specific species of insect. Furthermore, there may be trade-offs between some of these design features. For example, a trap with a large opening may have a greater number of insects entering the trap but also allow a greater number to escape. Some trap designs may have a high retention efficiency, i.e. number of insects contacting the trap compared to the number escaping, but suffer from a low maximum capacity. The differences in retention efficiency, maximum capacity and other trap features may have important implications on the effectiveness of trapping moths in high or low population densities or with highly attractive baits such as pheromones or less attractive baits such as kairomones.

Previous studies have found certain trap designs capture significantly more moths than other trap designs. For example Reardon et al. (2006) found large metal cone traps baited with pheromone to be the most effect trap compared to a small cone trap, Universal Moth Traps (UniTraps), and several sticky traps designed for catching male European corn borer, Ostrinia nubilalis (Hübner) (Crambidae). The authors concluded that the pheromone lures were attracting moths to all of the traps, but the insects were not sufficiently retained in the sticky traps; in addition they postulated that the larger diameter of the large cone traps played a role in greater captures compared to the small cone trap and UniTrap. In comparison, Knodel and Agnello (1990) found sticky traps caught higher numbers of Palpita unionlalis (Hübner) (Pyralidae) and other moth species than various funnel traps. However, the authors noted that moth populations were not high and because the sticky trap surfaces were changed weekly the chances of the traps becoming saturated with moths were reduced. For the noctuid species Spodoptera exigua (Höbner) (Noctuidae) funnel traps caught more moths than both sticky and cone traps (López Jr. 1998). 
Whitfield et al.: Comparison of different trap designs for capture of noctuid moths

There are numerous examples of how trap design affects catches of moths and non-targets using pheromone lures (e.g. López Jr. 1998, Maekawa et al. 1999, Matthews 1999, Reardon et al. 2006, Cha et al. 2011, Cork 2011, Guerrero et al. 2014), but little work has been carried out with trap designs baited with floral odors. Insects use very similar mechanisms to locate resources such as mates and food (Hardie et al. 2001, Schoonhoven et al. 2005), but there are obvious differences in how attractants based on these resources may work for use in traps. For noctuid moths traps baited with pheromone catch almost exclusively males. In contrast, floral odors are attractive to both males and females. The sensitivity of male noctuid moths to conspecific pheromones is extremely high, and males may be more sensitive to pheromone than female moths are to kairomones (Angioy et al. 2003). In addition, a trap baited with pheromone will generally experience less competition from the small amounts of pheromone produced by female moths than will a trap baited with floral odors from naturally released odors of flowers in the surrounding environment (Hardie et al. 2001). Consequently, traps baited with floral odors tend to capture fewer of the target individuals than pheromone-baited traps, and thus the retention efficiency of the trap becomes more important than the maximum number of insects the trap can contain. Therefore, when using floral odor attractants, or other kairomones, we might expect sticky traps to be more effective than other trap designs as sticky traps tend to have a larger surface area where insects can become ensnared by the sticky pad compared to the small surface area of a funnel (Athanassiou et al. 2007, Cardé et al. 2017).

Attractants based on floral volatiles not only attract both sexes of noctuid moths but also attract a wide range of other non-target species. This poses several problems such as removal of beneficial insects from the crop area, saturation of the trap with non-target insects potentially reducing the effectiveness of the trap, and confusion in identification of trapped insects for insect monitoring. Non-target captures can also be a problem even when using highly species specific pheromone lures and may be due to other cues such as vision (Knight and Miliczky 2003, Myers et al. 2009) or attraction of non-targets to the pheromone of the target species (Malo et al. 2001).

In this study, field trials were carried out to compare six trap types (five of which were tested in Argentina, and two tested in the United Kingdom) baited with either pheromone lures or a floral odor blend for the capture of various noctuid species. Although there are many reports of comparisons of trap designs baited with either pheromone or floral attractants, this study allowed direct comparison of the performance of traps with either type of lure, and the two 
Whitfield et al.: Comparison of different trap designs for capture of noctuid moths

different types of bait in the two crops allowed the traps to be compared whether there were many or few insects coming to the traps. In addition the numbers of non-target insects caught in the different designs of trap with the different lures are compared. 
Whitfield et al.: Comparison of different trap designs for capture of noctuid moths

\section{Materials and Methods}

\section{Lures}

Lures were prepared at the Natural Resources Institute (University of Greenwich, UK). Pheromone components were synthesized previously at NRI and were at least $97 \%$ pure by GC analysis. Components of the floral attractant were from Sigma Aldrich (Gillingham, Dorset, UK).

Pheromone lures for Autographa gamma (Linnaeus) were white rubber septa (International Pheromone Systems, Wirral, UK) impregnated with $0.1 \mathrm{mg}$ of (Z)-7-dodecenyl acetate and (Z)-7-dodecen-1-ol in a 10:1 ratio (Mazor and Dunkelblum 1992, 2005) and an equal amount of BHT as antioxidant.

Pheromone lures for Helicoverpa gelotopoeon (Dyar) were rubber septa impregnated with 1 mg of a 1:1 mixture of hexadecanal and (Z)-9-hexadecenal (Cork and Lobos 2003) and an equal amount of butylated hydroxytoluene (BHT) as an antioxidant.

The floral attractant lures contained phenylacetaldehyde (135 $\mu \mathrm{L}, \geq 95 \%)$, salicylaldehyde $(55 \mu \mathrm{L}, \geq 98 \%)$, methyl 2-methoxybenzoate (23.8 $\mu \mathrm{L}, \geq 97 \%)$, ( \pm )-linalool (23.8 $\mu \mathrm{L}, \geq 97 \%)$, and ( \pm )-limonene $(12.5 \mu \mathrm{L}, \geq 90 \%)$ (Cork 2011) on a cellulose acetate cigarette filter (14 x 6 mm, 14 x 6 mm, Swan, Republic Technologies Ltd., UK) in heat-sealed polyethylene sachets (50 mm x $50 \mathrm{~mm}, 120 \mu \mathrm{m}$ thick, Transatlantic Plastics, Southampton, UK). The sachets were shipped to Argentina in a coolbox, or stored at $-18{ }^{\circ} \mathrm{C}$ until used for the UK field trials.

\section{Traps}

The six trap types tested with their dimensions are shown in Fig. 1.

The sleeve trap (Pest Control India, Bangalore, India) consisted of a yellow plastic funnel with a green top over the funnel opening and a polyethylene sleeve coated with talcum powder to retain the insects that fell through the funnel. The lure was suspended beneath the lid. This trap costs approximately $£ 0.18 \mathrm{p} /$ unit.

The Universal Moth Trap (Unitrap; Agrisense, Treforest, UK), consisted of a yellow funnel within a white plastic bucket with a green lid. The lure was suspended from the lid in a 
Whitfield et al.: Comparison of different trap designs for capture of noctuid moths

plastic cage and insects entered through the funnel into the bucket. This trap costs approximately $£ 6.70$ / unit.

The wing trap (1C Wing Trap, IPM Technologies Inc., Portland, OR, USA) was a sticky trap with two halves held in place by a wire spacer, with a replaceable sticky sheet in the lower section of the trap. These traps cost approximately $£ 4.40$ / unit with sticky base.

The bucket trap (E. Lobos, Santiago Del Estero, Argentina) was made from a plastic bucket with lid and four holes cut in the sides, containing water with a layer of mineral oil to reduce surface tension and evaporation. The lure was suspended from the lid and insects entered the trap through the cut-out hole. The traps cost approximately $£ 0.50 \mathrm{p} /$ unit for the plastic materials, plus the time for cutting the holes.

The delta trap (AgriSense, Treforest, UK) was constructed from white Correx sheet with a replaceable sticky sheet on the base of the trap. These traps cost approximately $£ 5.00 /$ unit with sticky base.

The Lep trap (Plato Industries, Ltd., Houston, USA) was a white trap in two halves with a large sticky replaceable sheet on the bottom of the trap. The lure was positioned under the apex of the top section. These traps cost approximately $£ 9.50 /$ unit including multiple pheromone lures and six sticky bases.

\section{UK Field Trapping Experiments}

\section{Effect of Trap Type}

The trial compared catches of A. gamma male moths in the Unitrap and sleeve trap and was

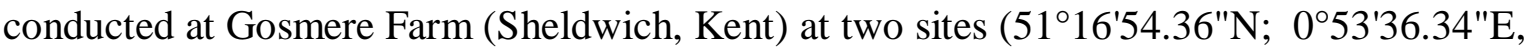
and $\left.51^{\circ} 16^{\prime} 19.90 " \mathrm{~N} ; 0^{\circ} 54^{\prime} 9.14 " \mathrm{E}\right)$. At one site the traps were position within $10 \mathrm{~m}$ of a crop of viper's buglos, Echium vulgare (L.) (Boraginaceae) at the late inflorescence stage. At the other site the traps were placed within $10 \mathrm{~m}$ of a crop of marigold, Tagetes erecta (L.) (Asteraceae) again in the inflorescent stage. Traps were placed in pairs with one UniTrap and one sleeve trap separated by $10 \mathrm{~m}$ around the fields at six locations. Catches were recorded at approximately 7-d intervals between June 182009 to August 232009 when the positions of each trap in the pair were exchanged. The daily temperatures throughout this period were: $11.1^{\circ} \mathrm{C}$ minimum $-23.8{ }^{\circ} \mathrm{C}$ maximum; rainfall was an average of $1.2 \mathrm{~mm}$ per day; sun hours 
Whitfield et al.: Comparison of different trap designs for capture of noctuid moths

were an average of $8.13 \mathrm{~h}$ per day. The pheromone lures for $A$. gamma were replaced once during the trial.

\section{Effect of Addition of Pesticide}

The trial assessed the effect on catches of moths of addition of a Vapona strip $(0.96 \%$ of Azamethiphos; $120 \mathrm{~mm}$ diameter; Ashe Ltd, UK) contact pesticide to the base of the bucket part of the Unitraps at the Echium vulgare and Tagetes fields at Gosmere Farm. Traps were baited with sachets containing the floral blend. There were four replicates of each treatment (pesticide present or not present) and the traps were positioned $10 \mathrm{~m}$ apart. The trial ran from August 4 to 23, 2009, and was positioned $100 \mathrm{~m}$ away from the trial investigating the effect of trap type. The daily temperatures throughout this period were: $14.1{ }^{\circ} \mathrm{C}$ minimum $-23.8{ }^{\circ} \mathrm{C}$ maximum; rainfall was an average of $0.45 \mathrm{~mm}$ per day; sun hours were an average of $8.0 \mathrm{~h}$ per day. Catches were counted six times with approximately 3-d intervals and positions of the two traps exchanged during this period.

\section{Argentina Field Trapping Experiments}

\section{Effects of Trap Type}

This trial compared the insect captures of five trap designs (the sleeve, bucket, wing, delta, and Lep traps) baited with either the sex pheromone of $H$. gelotopoeon or the floral odor blend. Two locations were used, one at a tomato field (2752'10.74"S; 6356'39.43"W) where the crop was at the unripe fruiting stage, and the second at the edge of a soybean field $\left(31^{\circ} 34^{\prime} 41.58^{\prime \prime} \mathrm{S} ; 63^{\circ} 43^{\prime} 46.13^{\prime \prime} \mathrm{W}\right)$ where the crop was in the third trifoliate stage. At each field site, two trials were run concurrently (approximately $100 \mathrm{~m}$ apart). One trial contained the five trap types with five replicates baited with $H$. geloptopoeon pheromone in a complete Latin square design with $10 \mathrm{~m}$ between the traps. This distance between traps was used to promote a degree of competition between the trap designs to enhance differences in performance, and to ensure that all traps in a replicate sampled the same population as far as possible. The other trial contained the five traps types with five replicates in a Latin square design, baited with the floral blend. Trap catches were counted and discarded once per week, i.e. three times between November 26 to December 102009 for the soybean trials; and once per week, i.e. four times between November 21 to December 132009 for the tomato trials. The average daily temperatures throughout this period were: $20.88^{\circ} \mathrm{C}$ average minimum, and $33.83{ }^{\circ} \mathrm{C}$ average maximum; rainfall was an average of $7.44 \mathrm{~mm}$ per day. After checking the 
Whitfield et al.: Comparison of different trap designs for capture of noctuid moths

traps were repositioned within their replicate and the insects removed or sticky sheets replaced. Numbers of $H$. geloptopoeon, other noctuids and other Lepidoptera were recorded. Non-target insects captured were classified in the field to genus or family if possible, or order. Species or genus identification was done by local entomology experts at the Facultad de Agronomia y Agroindustrias, Universidad Nacional de Santiago del Estero.

\section{Statistical Analyses}

Statistical analysis was done using R (R Core Team 2017) and R-Studio (RStudio Team 2016). The data from the UK field trials were analyzed by generalized linear model with a negative binomial distribution for the trap design trial and Poisson distribution for the addition of pesticide trial. Comparisons of the means were done using the 'glht' function from the MultComp package (Hothorn et al. 2008) for R.

The noctuid catch data from the Argentinian field trials were analyzed by generalized linear mixed effect model (GLMM) with a Poisson distribution and Date added as a random variable. In the initial model the following independent factors were considered: trap type, attractant type, crop type, and the interaction terms 'attractant:trap', 'attractant:crop', 'trap:crop', and 'attractant:trap:crop'. All factors were found to be significant (alpha level $p<$ 0.05). Following this, the data were split by crop (soybean and tomato) and attractant type (floral blend and sex pheromone of $H$. gelotopoeon), and analyzed by GLMM with trap type as the independent factor. Tukey's pair-wise comparisons were carried out with an alpha level of 0.05 using the MultComp package for R.

The non-target catch data was analyzed by GLMM with a negative binomial distribution and Date applied as a random variable. Data were split by attractant type.

\section{Results}

\section{UK Field Trials}

\section{Effect of Trap Type}

The initial field trial in the UK found a highly significant difference in the mean captures of male A. gamma caught in two trap types $\left(\chi_{(1,38)}^{2}=18.62, P<0.001\right)$. The funnel sleeve traps caught four times more moths than the Unitraps (Table 1). 
Whitfield et al.: Comparison of different trap designs for capture of noctuid moths

\section{Effect of Addition of Pesticide}

The moth captures with the floral blend were extremely low making statistical analysis difficult. Although there was a trend for a greater number of A. gamma to be caught in traps containing the pesticide compared to traps without pesticide, the difference was not significant (Table 2$)$. However, significantly more noctuid $\left(\chi_{(1, N=48)}^{2}=4.76, P<0.05\right)$ and total Lepidoptera $\left(\chi_{(1, N=48)}^{2}=4.55, P<0.05\right)$ were captured in the traps containing pesticide compared to untreated traps. Counts of A. gamma, Noctuidae and total Lepidoptera were more than double in traps containing the pesticide compared to traps without.

\section{Argentina Field Trials}

\section{Species of Noctuid Moths Caught}

The species of noctuid found and identified in the traps baited with the floral blend in the soybean trials included Helicoverpa zea (Boddie), Helicoverpa gelotopoeon (Dyar), Spodoptera frugiperda (J.E. Smith), Rachiplusia nu (Guenée), Dargida meridionalis (Hampson), and Mythimna either adultera (Schaus) or sequax (Franclemont) (unconfirmed). In the tomato trials, species identified were H. gelotopoeon, S. frugiperda, Chrysodeixis includens (Walker), and Agrotis either robusta (Blanchard) or malefida (Guenée) (unconfirmed). Catches of the individual species are shown in Table S1 of the Supplementary Material.

\section{Effect of Trap Type on Catches of Noctuid Moths}

For statistical analysis, captures of noctuid species were pooled. The traps baited with pheromone captured only males of $H$. gelotopoeon. The floral blend is a general attractant for noctuid moths, but the majority of moths caught in the traps baited with the floral blend were H. gelotopoeon (see Table S1 of the Supplementary Material).

The type of trap, attractant, crop, and the interaction terms were all found to be highly significant predictors of the number of noctuid moths caught (see Table S2 of the Supplementary Material). The date the traps were checked was found to have a significant effect on the counts of noctuid (see Fig. S1. of the Supplementary Material), and consequently was added to the model as a random variable. The data were subsequently split by crop (soybean and tomato) and attractant (floral blend and pheromone) (Fig. 2). The different trap types baited with the floral blend showed that the lowest number of noctuids were caught in the tomato crop, and the delta sticky trap captured significantly fewer 
Whitfield et al.: Comparison of different trap designs for capture of noctuid moths

individuals compared to bucket, sleeve, and wing traps. A moderate number of noctuids were caught in the traps baited with the floral blend in the soybean field. In this situation, the most effective trap type was the bucket $>$ sleeve $>$ LepTrap $>$ wing $=$ delta.

Traps baited with the pheromone caught significantly more noctuids than those baited with the floral blend, and with high trap catches the bucket trap caught significantly more noctuids than the sleeve trap in the tomato trial but not the soybean. Both of these traps caught significantly more noctuids compared to the three sticky trap types, with no significant differences between catches in the sticky traps (Fig. 2).

The traps baited with pheromone caught significantly more noctuids than the floral blend by about 6-fold. These were almost entirely H. gelotopoeon males in the pheromone-baited traps, but a mixture of male and female noctuid species in the traps baited with the floral blend.

\section{Non Target Captures}

A substantial number of non-target insects were captured in the traps, particularly in the traps baited with the floral blend (Fig. 3). Captures of beetles in three of the trap types, bucket, sleeve and delta, were particularly high in the traps baited with the floral blend, but were also found in traps baited with the H. geloptopoen pheromone. Insects of order Muscidae were also commonly found in the traps, regardless of the type of lure used. Several of the nontarget groups which were common in the traps baited with the floral blend were absent from the traps baited with the pheromone, i.e. Hymenoptera (other than wasps, bees, and parasitoids), Chrysopidae, and Diptera. For the traps baited with the floral blend in the soybean field, the bucket and sleeve traps caught significantly more non-target insects than the three sticky traps. These were predominantly Coleoptera.

\section{Discussion}

The hypothesis that sticky traps may be better traps in low target population situations (because of a larger capture area and therefore greater retention efficiency) and thus would be more effective when a kairomone attractant is used compared to a pheromone was not supported by these studies. The sticky traps tested in these trials consistently caught fewer moths compared to funnel and bucket traps even when their maximum capacity was not reached. The homemade bucket traps and the sleeve traps were better at capturing noctuid 
Whitfield et al.: Comparison of different trap designs for capture of noctuid moths

moths regardless of whether the bait was a highly attractive pheromone lure or a general floral attractant (kairomone).

The assessment of traps for use with kairomone lures is important, as these types of attractants have the potential to be extremely useful for pest population monitoring during pheromone-based mating disruption. Traps sold for population monitoring are often the 'sticky base' type traps, however, the results of this work suggest these may not be the most suitable trap design as they will capture fewer individuals. The major benefit to using 'sticky traps' is that the identification of the insects is easier than with funnel and bucket style traps, particularly if there are many non-targets captured as well.

Many previous studies comparing sticky traps to non-sticky traps show contradicting evidence. Some results showed non-sticky traps caught greater numbers of moths than sticky traps (e.g. Webster et al. 1986, Athanassiou et al. 2002, Athanassiou et al. 2004, Reardon et al. 2006), while others showed the contrary (e.g. Knodel and Agnello 1990, Athanassiou et al. 2007, Cardé et al. 2017). The reason for these confounding results is unclear, but certainly there will be differences due to factors other than the design of the traps, e.g. differences in behavior and size of the target insect, and also in population density. Authors have hypothesized that sticky traps may have a better retention efficiency than funnel traps because for the latter insects must fall through the funnel in order to be caught, whereas in a sticky trap the insect only has to contact the large sticky surface directly below the lure (Athanassiou et al. 2007). However, the sticky surface may quickly become saturated with debris and non-targets as well as the target insect. Therefore at low population densities the sticky trap may perform better due to a greater retention efficiency, but with larger population densities the funnel trap performs better as it has a much higher maximum capacity. In the present study we did not find that sticky traps performed better when fewer moths were coming to the traps, due to a less effective attractant rather than smaller moth population, which may suggest that the bucket-oil trap and the sleeve-funnel trap have a higher retention efficiency as well as a higher maximum capacity compared to the sticky-sheet based traps. UniTraps have been reported to be highly effective for capturing noctuids (López Jr. 1998) and had been used previously in numerous field work studies involving noctuids (e.g. Landolt et al. 2001, Meagher R.L 2001, Landolt and Higbee 2002, Camelo 2006, Meagher and Landolt 2010). However, the results from this study do not support their use for capturing noctuids. Comparison of the two funnel traps in the UK, the UniTrap and the sleeve trap, 
Whitfield et al.: Comparison of different trap designs for capture of noctuid moths

found that the sleeve trap caught significantly more A. gamma than the UniTrap. The opening of the funnel of both traps is the same size ( $90 \mathrm{~mm}$ diameter), but where the sleeve trap has a short (20 mm) funnel length and wide end $(45 \mathrm{~mm})$ the UniTrap has a much longer funnel (90 $\mathrm{mm})$ and smaller end $(30 \mathrm{~mm})$. Quite how much the shape of the funnel effects the capture efficiency is not known but we may assume that the longer funnel and smaller end of the UniTrap would make it more difficult for insects to escape from once they are in the bucket section below. The sleeve trap also contains an inert fine powder in the sleeve which may hinder an insect's ability to climb out of the sleeve by reducing friction. In addition, insects in the transparent sleeve will be subjected to desiccation from the sun whereas insects in the bucket of the UniTrap are shaded and may survive for longer allowing more escaping time. The addition of a contact pesticide doubled the number of noctuid moths caught in the UniTraps suggesting that one of the reasons for the trap's poor retention efficiency is that moths were able to escape back through the funnel. Other studies have included the use of killing agents in UniTraps but have suspected that their presence may also reduce the attractiveness of the trap to insects (Athanassiou et al. 2007) as they may be repelled by the vapors. In the present study a contact pesticide was used which should not have reduced the attractiveness of the trap.

The field trials in Argentina compared the previously tested sleeve trap with four other trap types: the homemade bucket and oil trap, and the three sticky traps. When the traps were baited with the floral blend fewer noctuid moths were found in the traps compared to baiting with the pheromone, but a wider variety of noctuid species were caught even though the majority were $H$. gelotopoeon. All of the noctuid species caught are considered crop pests to some degree. Although this bait was less effective in terms of absolute numbers of insects caught, it may prove useful to farmers for monitoring a wide range of crops pests with a single bait. However, the floral attractant baited traps also caught a larger number of nontarget insects compared to the pheromone baited traps. When baited with the floral attractant the captures of non-target insects in the traps followed a similar trend to that of the noctuids, i.e. the homemade bucket and the sleeve traps caught many more non-targets than the sticky traps. However, when baited with the pheromone, non-target insects were still caught, with significantly more in the delta trap compared to the LepTrap.

The two different types of bait in the two crops allowed the traps to be compared whether there were many or few insects coming to the traps. When a large number of insects are coming to the trap, maximum capacity is the key factor in determining the suitability of the 
Whitfield et al.: Comparison of different trap designs for capture of noctuid moths

trap; whereas if a small number of the insects are attracted to the trap, retention efficiency is the key factor in determining the trap catch. It was expected that the homemade bucket trap and the sleeve funnel trap would have the greatest capacity, but it may suffer from a lower retention efficiency due to the smaller surface area for capturing insects (approx. 13,200 $\mathrm{mm}^{2}$ for the bucket trap, and $6,300 \mathrm{~mm}^{2}$ for the sleeve trap). The sticky surface area of the three sticky traps were much larger than the bucket or sleeve trap: $32,300 \mathrm{~mm}^{2}$ for the LepTrap, 38,500 for the 'Wing', 32,300 $\mathrm{mm}^{2}$ for the delta, and it was expected that this larger surface area would convey an advantage in the capture of insects coming to the trap. However, we found that when the traps were baited with the floral blend there was either very little difference between the traps (in the tomato crop) or the bucket and sleeve traps outperformed the sticky traps (soybean crop).

Between the sticky traps the delta trap performed the worst as it caught significantly fewer noctuids than the LepTrap in the soybean trial and the wing trap in the tomato trial. Investigating three types of sticky traps (delta, wing, and diamond shaped), Knight et al. (2002) found that cumulative moth captures were proportional to the area of adhesive, and retention efficiency varied significantly between traps. Their research found the lowest retention was seen in wing traps which may have been because the flaps caused more moths to land on the outside of the trap compared to the other designs. Our data does not corroborate these findings.

When the traps were baited with the pheromone many more noctuids were caught in the traps, albeit almost exclusively only one species: H. gelotopoeon. These trials showed large significant differences between the bucket and sleeve traps compared to the three sticky traps in the number of noctuids caught. When the three sticky traps were baited with the sex pheromone they captured the same number of $H$. gelotopoeon (Fig. 2). This is similar to the results seen by Myers et al. (2009), who found no differences in captures of two pest Tortricid moths between LepTraps and delta sticky traps. The maximum capacity in these trials was approximately 50 noctuids / trap for the sticky traps, over 200 noctuids / trap for the sleeve traps, and almost 300 noctuids / trap for the homemade bucket trap.

The homemade bucket trap could be described as combining the best features of sticky traps and funnel traps. The oily layer on top of the water acting as a sticky surface trapping a high proportion of insects that come into contact with it, while the water filled area underneath can absorb large numbers of insects in the way that the bucket or sleeve of the funnel traps are 
Whitfield et al.: Comparison of different trap designs for capture of noctuid moths

able to. Thus, this bucket trap has a high retention proportion and also a high maximum capacity. Hot, dry weather can dry out the water in bucket traps (Diaz-Gomez et al. 2012), but in our trials the bucket traps had a thin layer of mineral oil on top of the water, which reduced aqueous evaporation.

The prices of the traps varied greatly, with sleeve trap and the homemade bucket trap being considerably cheaper (approximately $£ 0.18$ and $£ 0.50$ respectively) than the other traps (which ranged from $£ 4.40$ to $£ 9.50$ ). However, it should be noted that the durability of these traps may also vary, so consideration should be made for how long the traps may continue to be serviceable. Whilst the sleeve trap is the cheapest trap, it is also the most fragile and the plastic sleeve could be torn, especially in strong winds. The homemade bucket traps are also extremely cheap, but do require additional labor to cut the holes. Weather conditions may be a factor when considering which trap design to deploy. Excessive rain can flood traps making counting and identification of insects difficult, whilst excessive wind and dry dusty conditions can cause sticky traps to become saturated with debris. In our trials none of the traps suffered from negative effects of weather and all traps were in a good condition at the end of the trials.

No cone traps were evaluated in this study trials; although, previous work has shown that cone traps outperformed sleeve traps in capturing $H$. punctigera and $H$. armigera in New South Wales, Australia (Wilson and Morton 1989). Future work should investigate how the performance of cone traps compare to the homemade oil trap and sleeve trap, and what the retention ratio of these traps are. Future tests could include other design features such as trap color.

\section{Acknowledgments}

The authors would like to thank Maria Ana Laguzzi and Camilo Gómez Luengo for assistance running the trials in Argentina, and Dudley Farman of NRI for preparing the lures used throughout the trials. 
Whitfield et al.: Comparison of different trap designs for capture of noctuid moths

\section{References Cited}

Angioy, A. M., A. Desogus, I. T. Barbarossa, P. Anderson, and B. S. Hansson. 2003. Extreme sensitivity in an olfactory system. Chemical Senses 28: 279-284.

Athanassiou, C. G., N. G. Kavallieratos, and B. E. Mazomenos. 2004. Effect of trap type, trap color, trapping location, and pheromone dispenser on captures of male Palpita unionalis (Lepidoptera: Pyralidae). J. Econ. Entomol. 97: 321-329.

Athanassiou, C. G., N. G. Kavallieratos, F. T. Gravanis, N. A. Koukounitsas, and D. E. Roussou. 2002. Influence of trap type, pheromone quantity and trapping location on capture of the pink bollworm, Pectinophora gossypiella (Saunders) (Lepidoptera: Gelechiidae). Applied entomology and zoology 37: 385-391.

Athanassiou, C. G., N. G. Kavallieratos, S. F. Gakis, L. A. Kyrtsa, B. E. Mazomenos, and F. T. Gravanis. 2007. Influence of trap type, trap colour, and trapping location on the capture of the pine moth, Thaumetopoea pityocampa. Entomol. Exp. Appl. 122: 117-123.

Camelo, L. D. A. 2006. Floral Lures for Attract and Kill and for Seasonal Monitoring of Alfalfa Looper, Corn Earworm and Cabbage Looper Moths. Doctor of Philosophy, thesis, Washington State University.

Cardé, R. T., J. Bau, and J. S. Elkinton. 2017. Comparison of Attraction and Trapping Capabilities of Bucket- and Delta-Style Traps With Different Pheromone Emission Rates for Gypsy Moths (Lepidoptera: Erebidae): Implications for Understanding Range of Attraction and Utility in Surveillance. Environmental Entomology: nvx185-nvx185.

Cha, D. H., C. E. Linn, P. E. A. Teal, A. J. Zhang, W. L. Roelofs, and G. M. Loeb. 2011. Eavesdropping on Plant Volatiles by a Specialist Moth: Significance of Ratio and Concentration. Plos One 6: 8.

Cork, A. inventor UNIVERSITY OF GREENWICH, assignee. 2011. Insect attractant compositions. United Kingdom patent.

Cork, A., and E. A. Lobos. 2003. Female sex pheromone components of Helicoverpa gelotopoeon: First heliothine pheromone without (Z)-11-hexadecenal. Entomol. Exp. Appl. 107: 201-206.

Diaz-Gomez, O., E. A. Malo, S. A. Patiño-Arrellano, and J. C. Rojas. 2012. Pheromone Trap for Monitoring Copitarsia decolora (Lepidoptera: Noctuidae) Activity in Cruciferous Crops in Mexico. Florida Entomologist 95: 602-609.

Guerrero, S., J. Brambila, and R. L. Meagher. 2014. Efficacies of Four Pheromone-Baited Traps in Capturing Male Helicoverpa (Lepidoptera: Noctuidae) Moths in Northern Florida. Florida Entomologist 97: 1671-1678.

Hardie, J., G. Gibson, and T. D. Wyatt. 2001. Insect Behaviours Associated with Resource Finding. In I. P. R. Woiwod, D. R.; Thomas, C. D. (ed.), Insect Movement. CABI Publishing.

Hothorn, T., F. Bretz, and P. Westfall. 2008. Simultaneous Inference in General Parametric Models. Biometrical Journal 50: 17.

Knight, A. L., and E. Miliczky. 2003. Influence of Trap Colour on the Capture of Codling Moth (Lepidoptera: Tortricidae), Honeybees, and Non-target Flies. Journal of the Entomological Society of British Columbia. 100: 65-70.

Knight, A. L., D. Larson, and B. Christianson. 2002. Flight tunnel and field evaluations of sticky traps for monitoring codling moth (Lepidoptera: Tortricidae) in sex pheromone-treated orchards. Journal of the Entomological Society of British Columbia 99: 107-116.

Knodel, J. J., and A. M. Agnello. 1990. Field Comparison of Nonsticky and Sticky Pheromone Traps for Monitoring Fruit Pests in Western New York. J. Econ. Entomol. 83: 197-204.

Landolt, P. J., and B. S. Higbee. 2002. Both Sexes of the True Armyworm (Lepidoptera: Noctuidae) Trapped with the Feeding Attractant Composed of Acetic Acid and 3-Methyl-1-Butanol. The Florida Entomologist 85: 182-185. 
Whitfield et al.: Comparison of different trap designs for capture of noctuid moths

Landolt, P. J., T. Adams, H. C. Reed, and R. S. Zack. 2001. Trapping alfalfa looper moths (Lepidoptera : Noctuidae) with single and double component floral chemical lures. Environmental Entomology 30: 667-672.

López Jr., J. D. 1998. Evaluation of Some Commercially Available Trap Designs and Sex Pheromone Lures for Spodoptera exigua (Lepidoptera: Noctuidae). J. Econ. Entomol. 91: 517-521.

Maekawa, M., T. Imai, S. Tsuchiya, T. Fujimori, and W. S. Leal. 1999. Behavioral and electrophysiological responses of the soybean beetle, Anomala rufocuprea Motschulsky (Coleoptera : Scarabaeidae) to methyl anthranilate and its related compounds. Applied entomology and zoology 34: 99-103.

Malo, E. A., L. Cruz-Lopez, J. Valle-Mora, A. Virgen, J. A. Sanchez, and J. C. Rojas. 2001. Evaluation of Commercial Pheromone Lures and Traps for Monitoring Male Fall Armyworm (Lepidoptera: Noctuidae) in the Coastal Region of Chiapas, Mexico. The Florida Entomologist 84: 659-664.

Matthews, M. 1999. Heliothine moths of Australia : a guide to pest bollworms and related noctuid groups / Marcus Matthews, CSIRO Publishing, Melbourne :.

Mazor, M., and E. Dunkelblum. 1992. Role of sex pheromone components in behavioral reproductive isolation between Autographa gamma (L.) and either Trichoplusia ni (Hübner) OR Chrysodeixis chalcites (Esp.) (Lepidoptera: Noctuidae: Plusiinae). Journal of Chemical Ecology 18: 2373-2384.

Mazor, M., and E. Dunkelblum. 2005. Circadian rhythms of sexual behavior and pheromone titers of two closely related moth species Autographa gamma and Cornutiplusia circumflexa. Journal of Chemical Ecology 31: 2153-2168.

Meagher R.L, Jr. 2001. Collection of Fall Armyworm (Lepidoptera: Noctuidae) adults and nontarget Hymenoptera in different colored Unitraps. Florida Entomologist 84: 77-82.

Meagher, R. L., and P. J. Landolt. 2010. Binary Floral Lure Attractive to Velvetbean Caterpillar Adults (Lepidoptera: Noctuidae). Florida Entomologist 93: 73-79.

Myers, C. T., G. Krawczyk, and A. M. Agnello. 2009. Response of tortricid moths and non-target insects to Pheromone trap colour in commercial apple orchards. Journal of Entomological Science 44: 69-77.

R Core Team 2017. R: A language and environment for statistical computing. computer program, version 3.4.2. By R Core Team, Vienna, Austria.

Reardon, B. J., D. V. Sumerford, and T. W. Sappington. 2006. Impact of trap design, windbreaks, and weather on captures of European Corn Borer (Lepidoptera: Crambidae) in pheromonebaited traps. J. Econ. Entomol. 99: 2002-2009.

RStudio Team 2016. RStudio: Integrated Development for R computer program, version 1.1.383. By RStudio Team, Boston, MA.

Schoonhoven, L. M., J. J. A. van Loon, and M. Dicke. 2005. Host-plant selection: how to find a host plant. In L. M. Schoonhoven, J. J. A. van Loon and M. Dicke (eds.), Insect-Plant Biology, 2nd ed. Oxford University Press, Oxford, UK.

Webster, R. P., R. E. Charlton, C. Schal, and R. T. Cardé. 1986. High-efficiency Pheromone Trap for the European Corn Borer (Lepidoptera: Pyralidae). J. Econ. Entomol. 79: 1139-1142.

Wilson, A. G. L., and R. Morton. 1989. Some factors affecting the reliability of pheromone traps for measurement of the relative abundance of Helicoverpa punctigera (Wallengren) and $\mathrm{H}$. armigera (Hubner) (Lepidoptera: Noctuidae). Bulletin of Entomological Research 79: 265273. 
Whitfield et al.: Comparison of different trap designs for capture of noctuid moths

\section{Tables}

Table 1 Mean catches of Autographa gamma male moths caught in two types of traps baited with pheromone during UK field trial between June 18 and August 23, 2009. Means are captures over the total trapping period, values in parentheses show standard error, and different letters denote significant differences between the treatments $(P<0.05)$. Data analyzed by GLM with negative binomial distribution and Tukey's pairwise comparisons.

\begin{tabular}{lll} 
Trap type & $N$ & Mean captures male Autographa gamma (SE) \\
\hline UniTrap & 10 & $1.75(0.44) a$ \\
Sleeve trap & 10 & $7.15(1.27) b$ \\
\hline
\end{tabular}

Table 2 Mean catches of Autographa gamma (males and females), total Noctuidae, and total Lepidotpera caught in UniTraps baited with the floral blend, with or without pesticide (Azamethiphos) in the traps in UK (4-23 August 2009). Values in parentheses show standard error, and different letters denote significant differences between the treatments $(P<0.05)$. Data analyzed by chi-squared test.

\begin{tabular}{lllll}
\hline \multirow{4}{*}{ Pesticide } & $N$ & \multicolumn{2}{l}{ Mean catches (SE) } \\
\cline { 3 - 5 } & $N$ & Autographa gamma & Noctuidae & Lepidoptera \\
\hline None & 24 & $0.13(0.07)$ & $0.17(0.08) a$ & $0.25(0.09) a$ \\
Present & 24 & $0.25(0.11)$ & $0.54(0.26) b$ & $0.67(0.25) b$ \\
\hline
\end{tabular}


Whitfield et al.: Comparison of different trap designs for capture of noctuid moths

\section{Figures}

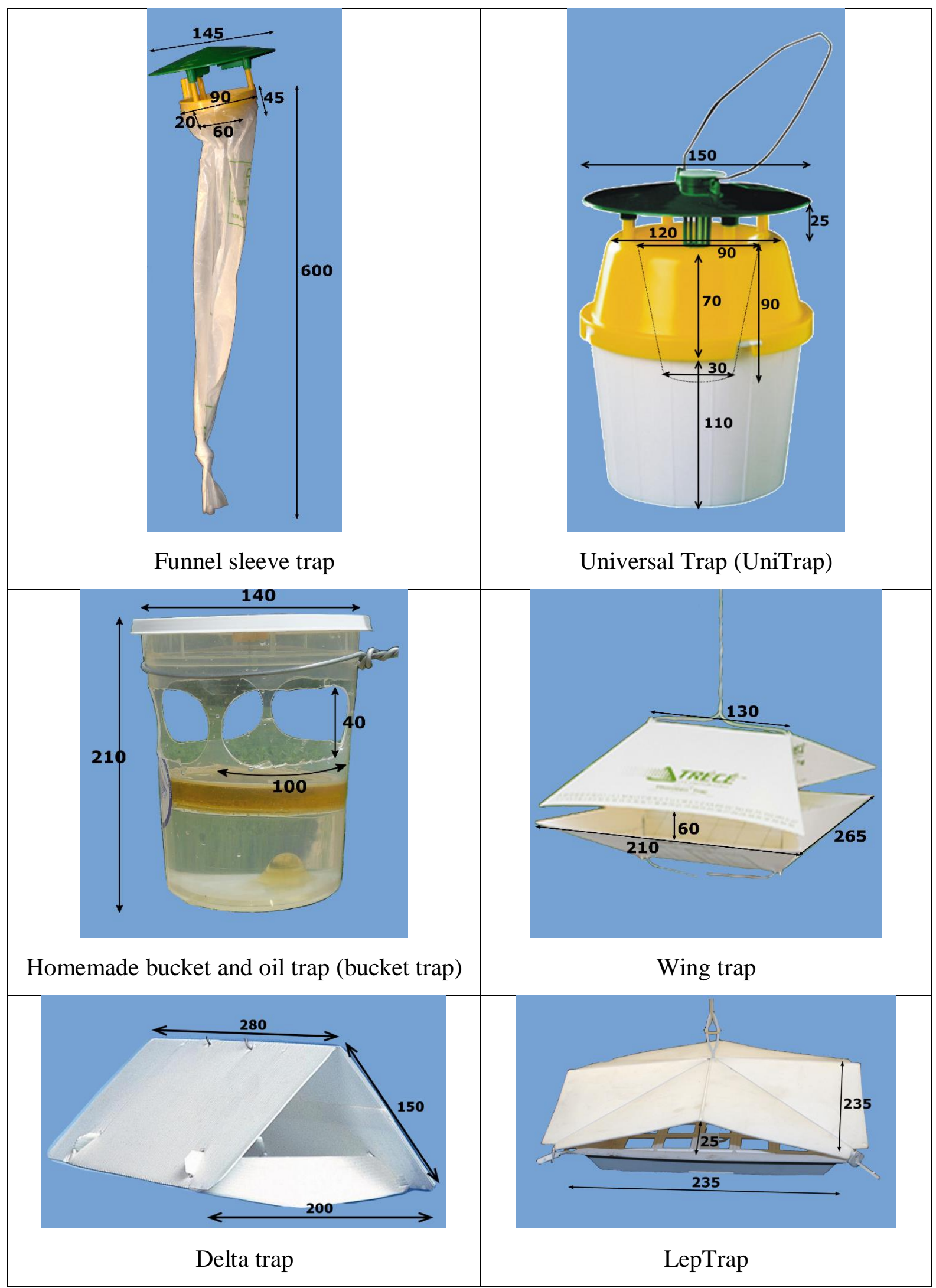

Figure 1: Traps used in the field trials with dimensions in $\mathrm{mm}$. Descriptions in the main text. 
Whitfield et al.: Comparison of different trap designs for capture of noctuid moths

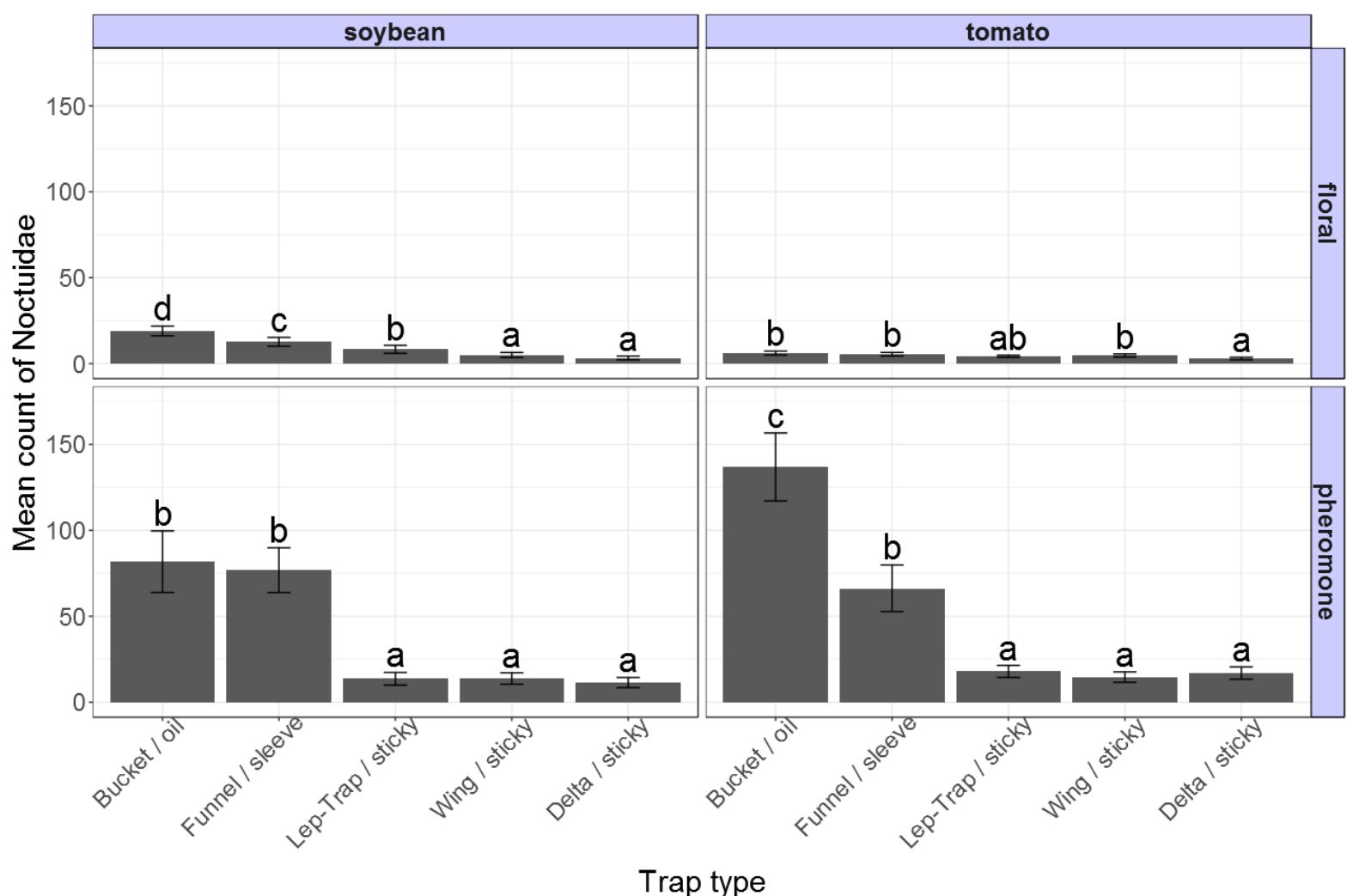

Figure 2: Mean number of noctuid moths caught in five traps baited with either a floral odor blend or sex pheromone of Helicoverpa gelotopoeon in soybean and tomato field trials. The error bars show standard error of the mean. Different letters denote a significant difference between traps within the same chart. Data analysed by Generalised linear mixed-effect model (GLMM) with a Poisson distribution and 'date' applied as a random variable. $N=15$ for the soybean trials and 20 for the tomato trials. 
Whitfield et al.: Comparison of different trap designs for capture of noctuid moths

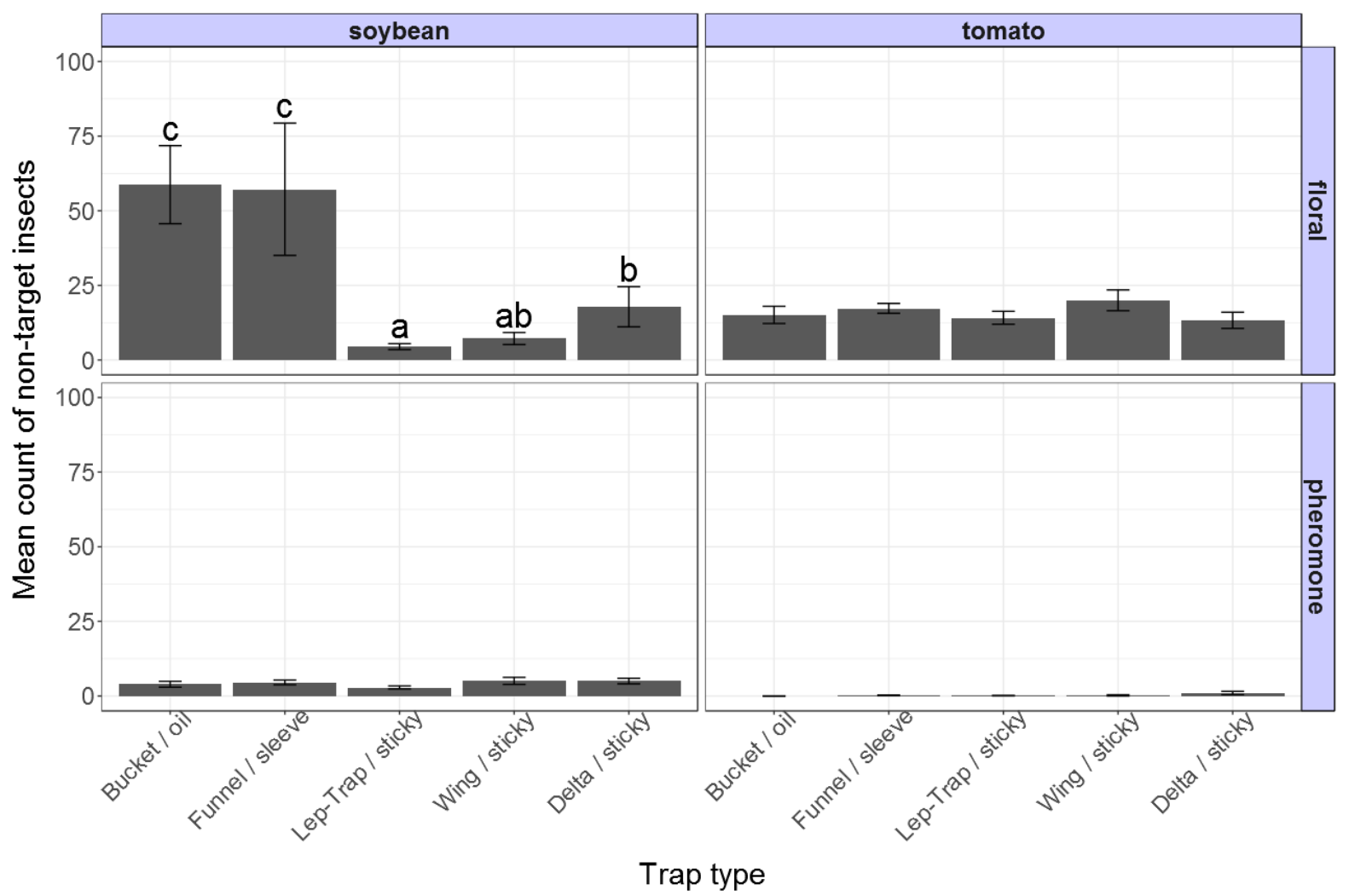

Figure 3: Mean numbers of non-target insects caught in five traps baited with either a floral blend or sex pheromone of Helicoverpa gelotopoeon in soybean and tomato field trials in Argentina. The error bars show standard error of the mean. Different letters denote a significant difference between traps within the same chart. Data were analyzed by generalized linear mixed-effect model (GLMM) with a negative binomial distribution and 'date' applied as a random variable. $N=15$ for the soybean trials and 20 for the tomato trials. 